Copyright (C) 2013 IEEE. Personal use of this material is permitted. Permission from IEEE must be obtained for all other uses, in any current or future media, including reprinting/republishing this material for advertising or promotional purposes, creating new collective works, for resale or redistribution to servers or lists, or reuse of any copyrighted component of this work in other works. 


\title{
On the definition of negative imaginary system for not necessarily rational symmetric transfer functions*
}

\author{
Augusto Ferrante ${ }^{\dagger}$ and Lorenzo Ntogramatzidis ${ }^{\star}$
}

\begin{abstract}
In this paper we provide a definition and characterisation of negative imaginary systems for not necessarily rational but symmetric transfer functions along the same lines of the classic definition of positive real systems. We then derive a necessary and sufficient condition that characterises symmetric negative imaginary transfer functions in terms of a matrix sign condition on the imaginary axis.
\end{abstract}

\section{INTRODUCTION}

The notion of positive real system was first introduced in electrical network analysis to indicate dissipative $m$-port networks. In the single-input single-output case, positive real systems can be realised with electrical circuits involving only resistors, capacitors and inductors. In the multipleinput multiple-output case, $m$-port electrical networks can be realised using also transformers and gyrators, in addition to resistors, capacitors and inductor. The most interesting case is by far the one of the so-called reciprocal m-ports, which can be realised by the above mentioned electrical components with the exception of gyrators. The interesting feature of reciprocal $m$-port electrical networks is the fact that the corresponding impedance, admittance and scattering matrices are always symmetric. Furthermore, in practice gyrators can only be realised by using active electrical elements. We refer to [1] and the references cited therein.

A fundamental feature of the classic positive real theory is the fact that the relative degree of rational positive real systems is either zero or \pm 1 , [2]. It was noticed in [15] that, when modelling undamped or lightly damped flexible structures with colocated position sensors and force actuators, the resulting transfer function has a relative degree which is greater than one. This consideration led to the introduction of the so-called negative imaginary systems, which impose a weaker restriction on the relative degree of the transfer function. A very promising stream of literature flourished in the last few years on the properties of negative imaginary systems, see [16], [22], [23], [21], [20], [19], [17], [24] and the references cited therein.

In the first contribution on this topic [15], a definition was proposed for negative imaginary function matrix in terms

*Partially supported by the Italian Ministry for Education and Research (MIUR) under PRIN grant n. 20085FFJ2Z "New Algorithms and Applications of System Identification and Adaptive Control" and by the Australian Research Council under the grant FT120100604.

†Augusto Ferrante is with Dipartimento di Ingegneria dell'Informazione, Università di Padova, via Gradenigo, 6/B - 35131 Padova, Italy. augusto@dei.unipd.it

*Lorenzo Ntogramatzidis is with the Department of Mathematics and Statistics, Curtin University, Perth (WA), Australia. L. Ntogramatzidis@curtin.edu.au of properties of the function on the imaginary axis. In this first definition, systems with poles on the imaginary axis were excluded, see also the contribution by the same authors in IEEE Control Systems Magazine, [19]. This restriction was abandoned in [23], [21], where a simple pole on the imaginary axis except at the origin was allowed. A further important extension of this new negative imaginary theory has subsequently been proposed to deal with systems with a single pole at the origin, [17]. More recently, also the case of a double pole at the origin was considered, see [14], [18]. In the past few years, several efforts have been devoted to the generalisation and adaptation of this theory to different contexts, including implicit systems in [17]. Lossless imaginary systems have also been recently introduced in [22] and [24]. Systems with two poles at the origin have also been recently considered in [18].

The reason for the success of the negative imaginary theory is that many systems that are important and useful in practice are negative imaginary.

The aim of this paper is to provide a definition of negative imaginary system for a transfer function which does not necessarily correspond to a finite-dimensional realisation. Such definition is given in terms of a matrix sign condition in the entire domain of analyticity, in the same spirit of the classic positive real case. Our effort in this direction was motivated by the results on the closed loop stability of systems having a (non necessarily rational) positive real transfer function.

Then, in a way that parallels the conventional positive real approach, [1], [2], a necessary and sufficient condition will be introduced for the rational case that characterises a negative imaginary symmetric transfer function in terms of its behaviour on the imaginary axis. This alternative approach has the advantage of being applicable to possibly non-proper transfer functions, as well as to transfer functions with more than one pole at the origin, and even to non rational transfer functions. The price to pay is the restriction to symmetric transfer functions. Nevertheless, as aforementioned, the symmetric case is by far the most relevant and interesting in practice, as it is the one that corresponds to reciprocal $m$-port electrical networks. In addition, undamped or lightly damped flexible structures with colocated position sensors and force actuators are characterised by a symmetric transfer function, [19], [24].

In this paper, we also analyse the connections between negative imaginary and positive real rational functions. This is of crucial importance since positive real functions and the celebrated Positive Real Lemma (see [5], [6] for two fairly 
recent papers whose reference lists points to many of the fundamental contributions and generalisations in this field). We also recall that Positive Real Lemma may be viewed as the "father" of the algebraic Riccati equation (ARE) which plays a fundamental role in almost any area of systems theory, both in the discrete in the continuos time cases, see e.g. [8], [9], [3], [7], [10], [4], [13], [12] for a sample of different aspects, properties and applications of ARE and for an extensive reference list.

This is the conference version of the journal paper [11] submitted by the same authors to Automatica and currently in press.

Notation. Given a matrix $A, A^{\mathrm{T}}$ denotes the transpose of $A$ and $A^{*}$ denotes the complex conjugate transpose of $A$. Given a matrix-valued function $F(s)$, we denote by $F^{*}(s)$ the function $[F(s)]^{*}$.

\section{Positive Real and Negative Imaginary FUNCTIONS}

We begin by recalling the classical definition of positive real function. For a detailed discussion on positive real systems we refer to [1], [2] and the references cited therein.

Definition 2.1: [Positive Real Function]

The function $F: \mathbb{C} \longrightarrow \mathbb{C}^{m \times m}$ is positive real if

- $F(s)$ is analytic in $\mathfrak{R e}\{s\}>0$;

- $F(s)$ is real for all positive real $s$;

- $F(s)+F^{*}(s)$ is positive semidefinite for all $\mathfrak{R e}\{s\}>0$.

For a detailed discussion on positive real systems we refer to the important textbooks [1], [2].

The following classic result provides necessary and sufficient conditions for a real rational transfer function matrix to be positive real in terms of the behaviour of its restriction to the imaginary axis of the complex plane.

Lemma 2.1: Let $F(s)$ be real and rational. Then $F(s)$ is positive real if and only if

- $F(s)$ has no poles in $\mathfrak{R e}\{s\}>0$;

- $F(i \omega)+F^{*}(i \omega) \geq 0$ for all real $\omega$ such that $i \omega$ is not a pole of $F(i \omega)$;

- if $s=i \omega_{0}$ is a pole of any element of $F(s)$, it is a simple pole and the corresponding residual is a semidefinite Hermitian matrix.

We now present a definition of negative imaginary system for transfer functions which are not necessarily rational. In this definition, similarly to what happens for the classic positive real case, the sign condition holds in the entire domain of analyticity of the transfer function. We recall that here our attention is restricted to symmetric matrix transfer functions. We begin by giving a definition of skew-imaginary transfer function.

Definition 2.2: [SKEW-IMAGINARY FUnCTION]

Let $G: \mathbb{C} \longrightarrow \mathbb{C}^{m \times m}$ be analytic in an open subset $\Omega$ of $\mathbb{C}$. Then, $G$ is skew-imaginary in $\Omega_{1} \subseteq \Omega$ if

- $i\left[G(s)-G^{*}(s)\right] \geq 0$ for all $s \in \Omega_{1}$ such that $\mathfrak{I m}\{s\}>0$;
- $i\left[G(s)-G^{*}(s)\right]=0$ for all $s \in \Omega_{1}$ such that $\mathfrak{I m}\{s\}=0$;

- $i\left[G(s)-G^{*}(s)\right] \leq 0$ for all $s \in \Omega_{1}$ such that $\mathfrak{I m}\{s\}<0$.

Definition 2.3: [SYMmetric Negative ImaginaRy FUNCTION]

Let $G: \mathbb{C} \longrightarrow \mathbb{C}^{m \times m}$ be a real, symmetric transfer function. We say that $G$ is symmetric negative imaginary if

- $G(s)$ is analytic in $\mathfrak{R e}\{s\}>0$;

- $G(s)$ is skew-imaginary in $\mathfrak{R e}\{s\}>0$.

Since $G(s)$ is symmetric, $i\left[G(s)-G^{*}(s)\right]=-2 \mathfrak{I m}\{G(s)\}$.

As noted above, with respect to the definitions of negative imaginary transfer functions given in [15], [17] and [21], here we restrict our attention to the symmetric ones. On the other hand, a definition given directly in terms of a sign property of the transfer matrix function in its domain of analyticity appears to be very appealing since it goes in the direction of an abstract foundation in the same spirit of the definition of positive real functions. In particular, in this definition, there is no need to start with rational and proper functions as in [15], [17] and [21] or to deal with poles. Notice that this definition may immediately be applied to non-proper rational transfer functions. For example, consider $G(s)=a s$, where $a \in \mathbb{R}$. Then, $G(s)$ is analytic in $\mathfrak{R e}\{s\}>0$ and, by defining $s=\sigma+i \omega$, we get

$$
i\left[G(s)-G^{*}(s)\right]=i[a \sigma+i a \omega-(a \sigma-i a \omega)]=-2 a \omega,
$$

which is non-negative for all $\omega \geq 0$ if and only if $a \leq 0$. It follows that $G(s)$ is negative imaginary if and only if $a \leq 0$.

Moreover, the fact, introduced in [14] as an ad hoc extension, that a transfer function with a double pole in the origin may be negative imaginary is also a direct consequence of this general definition. For example, $G(s)=1 / s^{2}$ is easily seen to be negative imaginary. Indeed, defining $s=\sigma+i \omega$, in the domain of analyticity $(\sigma>0)$ the quantity

$$
i\left[G(s)-G^{*}(s)\right]=\frac{4 \sigma \omega}{\left(\sigma^{2}-\omega^{2}\right)^{2}+4 \sigma^{2} \omega^{2}}
$$

is positive definite for all $\omega \geq 0$.

Finally, to the best of our knowledge, this is the first definition that allows the notion of negative imaginary system to be applicable to non-rational transfer functions. For example, consider a system with a delay described by the transfer function $G(s)=-s\left(e^{-s T}+1\right)$, with $T \in \mathbb{R}_{+}$. We now show that $G(s)$ is negative imaginary. Let $s=\sigma+i \omega$. A direct calculation yields

$i\left(G(s)-G^{*}(s)\right)=2\left[\omega+e^{-\sigma T}(\omega \cos (\omega T)-\sigma \sin (\omega T))\right]$.

Now define

$$
f(\sigma, \omega) \stackrel{\text { def }}{=} \omega+e^{-\sigma T}(\omega \cos (\omega T)-\sigma \sin (\omega T)) .
$$

Since $f(\sigma, 0)=0$ for all $\sigma \geq 0$, and $f(\sigma,-\omega)=-f(\sigma, \omega)$, we only need to show that, when $\sigma>0$ and $\omega>$ $0, f(\sigma, \omega) \geq 0$. Assume by contradiction that $\bar{\sigma} \geq 0$ and $\omega_{0}>0$ exist such that $f\left(\bar{\sigma}, \omega_{0}\right)<0$. It is immediate to see that $f\left(\sigma, \omega_{0}\right)$, as a function of $\sigma$, is continuos with all its derivatives. Hence, there exists $\sigma_{0}$ 
such that $\inf _{\sigma \geq 0} f\left(\sigma, \omega_{0}\right)=\min _{\sigma \geq 0} f\left(\sigma, \omega_{0}\right)=f\left(\sigma_{0}, \omega_{0}\right) \leq$ $f\left(\bar{\sigma}, \omega_{0}\right)<0$ and $\frac{\partial f}{\partial \sigma}\left(\sigma_{0}, \omega_{0}\right)=0$. By computing the derivative

$$
\frac{\partial f}{\partial \sigma}\left(\sigma, \omega_{0}\right)=e^{-\sigma T}\left[\sigma T \sin \left(\omega_{0} T\right)-\sin \left(\omega_{0} T\right)-\omega_{0} T \cos \left(\omega_{0} T\right)\right]
$$

we see that the only candidate $\sigma_{0}$ is given by

$$
\sigma_{0}=\frac{1}{T}+\omega_{0} \frac{\cos \left(\omega_{0} T\right)}{\sin \left(\omega_{0} T\right)} .
$$

To get a contradiction, it is now sufficient to show that if $\omega_{0}$ is such that $\sigma_{0}$ is finite and positive, then $f\left(\sigma_{0}, \omega_{0}\right)>$ 0 . Indeed, $f\left(\sigma_{0}, \omega_{0}\right)=\frac{1}{T}\left[\omega_{0} T-e^{-\sigma_{0} T} \sin \left(\omega_{0} T\right)\right]$, which is clearly positive for all $\omega_{0}>0, \sigma_{0}>0$ and $T>0$. In fact, if $\sin \left(\omega_{0} T\right) \leq 0$, this is obvious, while if $\sin \left(\omega_{0} T\right)>0$, we have

$$
\frac{1}{T}\left[\omega_{0} T-e^{-\sigma_{0} T} \sin \left(\omega_{0} T\right)\right] \geq \frac{1}{T}\left[\omega_{0} T-\sin \left(\omega_{0} T\right)\right]
$$

which is positive for all $\omega_{0} T>0$.

\section{NEGATIVE IMAGINARY REAL-RATIONAL TRANSFER FUNCTIONS}

In this section, we now specialise the notion of symmetric function to real and rational transfer function matrices. We begin by recalling that given a real rational function $G(s)$ and a simple pole $p \in \mathbb{C}$ of $G(s)$, we have a unique decomposition

$$
G(s)=G_{1}(s)+\frac{A}{(s-p)},
$$

where $G_{1}(s)$ is a rational function which is analytic in an open set containing $p$ and the (non-zero) matrix $A$ is the residue corresponding to the pole $p$. If $p$ is a double pole of $G(s)$, we have the unique decomposition

$$
G(s)=G_{1}(s)+\frac{A_{1}}{s-p}+\frac{A_{2}}{(s-p)^{2}},
$$

where the matrix $A_{1}$ is the residue corresponding to the pole $p$. In this case, by analogy, we define the (non-zero) matrix $A_{2}$ to be the quadratic residue corresponding to the pole $p$. If $G(s)$ has a pole at infinity, it can be uniquely decomposed as

$$
G(s)=G_{1}(s)+P(s),
$$

where $G_{1}(s)$ is a rational proper function and $P(s)=\sum_{i=1}^{k} A_{i} s^{i}$ is a homogeneous polynomial in $s$. We refer to $A_{i}$ as the $i$-th coefficient in the expansion at infinity of $G(s)$.

Lemma 3.1: Let $G(s)$ be real, symmetric and rational. Then $G(s)$ is symmetric negative imaginary if and only if

(i) $G(s)$ has no poles in $\mathfrak{R e}\{s\}>0$;

(i) $i\left[G(i \omega)-G^{*}(i \omega)\right] \geq 0$ for all $\omega \in(0, \infty)$ except for the values of $\omega$ where $i \omega$ is a pole of $G(s)$;

(iii) if $s=i \omega_{0}$, with $\omega_{0} \in(0, \infty)$, is a pole of $G(s)$, it is a simple pole and the corresponding residual matrix $^{1} K_{0}=\lim _{s \rightarrow i \omega_{0}}\left(s-i \omega_{0}\right) i G(s)$ is Hermitian and positive semidefinite;

\footnotetext{
${ }^{1}$ Notice that $K_{0}$ is the product of the imaginary unit $i$ by the residue in
}

(iv) if $s=0$ is a pole of $G(s)$, it is at most a double pole. Moreover, both its residual and its quadratic residual (when present) are positive semidefinite Hermitian matrices;

(v) if $s=\infty$ is a pole of $G(s)$, it is at most a double pole. Moreover, both the coefficients in the expansion at infinity of $G(s)$ are negative semidefinite Hermitian matrices.

A complete proof of this result can be found in [11], proof of Lemma 3.1.

The following result is crucial in the characterisation of the relationship between negative imaginary and positive real rational functions.

Lemma 3.2: $F(s)$ is a real rational positive real function if and only if it may be written as

$$
F(s)=(1 / s) A+F_{1}(s)
$$

where $A$ is symmetric and positive semidefinite and $F_{1}(s)$ is a real rational positive real function with no poles at the origin.

Proof: If $F(s)$ has the form (1) then it is clearly positive real in view of Lemma 2.1. Conversely, assume that $F(s)$ is positive real. If $F(s)$ has no poles at the origin, we may take $F_{1}(s)=F(s)$ and $A=0$ and we have the required decomposition. If $F(s)$ has a pole at the origin, this pole is simple and with symmetric and positive definite residue $A$, in view of Lemma 2.1. Then, we can decompose $F(s)$ in the form (1) where $A$ is indeed the residue at the origin and has therefore the prescribed properties and $F_{1}(s)$ is a real rational function without poles at the origin. It remains to show that $F_{1}$ is itself positive real. To this end, we use again Lemma 2.1. Since $F(s)$ is positive real, it is analytic on the open right half-plane and hence $F_{1}(s)$ has the same property. Moreover, if $i \omega_{0}$ is a pole of $F_{1}(s)$ with multiplicity $\mu$ and residue $A_{0}$, then it is also a pole of $F(s)$ with the same multiplicity and the same residue. Then, $\mu=1$, and $A_{0}$ is symmetric and positive semidefinite. The same holds for a possible pole of $F_{1}(s)$ at infinity. Finally, notice that for $\omega \neq 0$ such that $i \omega$ is not a pole of $F_{1}$ we have $F(i \omega)+F^{*}(i \omega)=F_{1}(i \omega)+F_{1}^{*}(i \omega)$ so that $F_{1}(i \omega)+F_{1}^{*}(i \omega)$ is Hermitian and positive semidefinite. Since $F_{1}(s)+F_{1}^{*}(s)$ is analytic at the origin, we also have that $F_{1}(0)+F_{1}^{*}(0)$ is Hermitian and positive semidefinite.

The following theorem is is the analogous of Lemma 3 in [21], but valid under the definition of symmetric negative imaginary matrix function given here.

Theorem 3.1: Let $G(s)$ be a real rational and proper symmetric negative imaginary matrix transfer function. Then $F(s) \stackrel{\text { def }}{=} s[G(s)-G(\infty)]$ is positive real. Conversely, let $F(s)$ be symmetric, real rational and positive real matrix transfer function. Then $G(s) \stackrel{\text { def }}{=}(1 / s) F(s)+D$ is symmetric negative imaginary for any symmetric matrix $D$. 
Proof: Let $G(s)$ be a real rational and proper symmetric negative imaginary matrix transfer function. We have to show that $F(s) \stackrel{\text { def }}{=} s[G(s)-G(\infty)]$ is positive real. It is clear that $F(s)$ is analytic in the open right half plane and has no pole at infinity. Moreover, $F$ and $G$ have the same poles with the possible exception of the pole at the origin. Let $\omega_{0}>0$ be given and assume that $s=i \omega_{0}$ is not a pole of $G(s)$. Then, it is not a pole of $F(s)$ and, since $G^{*}(\infty)=G^{\mathrm{T}}(\infty)=G(\infty)$, we find

$$
\begin{aligned}
F\left(i \omega_{0}\right) & +F^{*}\left(i \omega_{0}\right) \\
& =i \omega_{0}\left[G\left(i \omega_{0}\right)-G(\infty)\right]-i \omega_{0}\left[G^{*}\left(i \omega_{0}\right)-G^{*}(\infty)\right] \\
& =i \omega_{0}\left[G\left(i \omega_{0}\right)-G^{*}\left(i \omega_{0}\right)\right]+i \omega_{0}\left[G^{\mathrm{T}}(\infty)-G(\infty)\right] \\
& =\omega_{0}\left(i\left[G\left(i \omega_{0}\right)-G^{*}\left(i \omega_{0}\right)\right]\right) \geq 0,
\end{aligned}
$$

where the last inequality holds since $\omega_{0}>0$ and $G$ is symmetric negative imaginary. Assume now that $\omega_{0}>0$ is such that $s=i \omega_{0}$ is a pole of $G(s)$. Then, it is necessarily a simple pole of $G(s)$, and it is also a simple pole of $F(s)$. We then have $G(s)=\frac{A}{s-i \omega_{0}}+G_{1}(s)$ and $K_{0} \stackrel{\text { def }}{=} i A$ is Hermitian and positive semidefinite. Let us compute the residue of $F(s)$ in $i \omega_{0}$. This is given by

$$
\begin{aligned}
& \lim _{s \rightarrow i \omega_{0}}\left(s-i \omega_{0}\right) F(s)= \\
& \quad \lim _{s \rightarrow i \omega_{0}} s A+\left(s-i \omega_{0}\right)\left[G_{1}(s)-G(\infty)\right]=\omega_{0} i A \geq 0 .
\end{aligned}
$$

Let us consider now the case when $\omega_{0}<0$. If $s=i \omega_{0}$ is not a pole of $G(s)$ and $F(s)$, we have $F\left(i \omega_{0}\right)+F^{*}\left(i \omega_{0}\right)=$ $\overline{F\left(-i \omega_{0}\right)+F^{*}\left(-i \omega_{0}\right)}$ which is positive semidefinite since it is the complex conjugate of $F\left(-i \omega_{0}\right)+F^{*}\left(-i \omega_{0}\right)$, and this has already been seen to be positive semidefinite. A similar argument can be used to show that when $s=i \omega_{0}$ is a pole of $G(s)$ and $F(s)$, its residue as a pole of $F$ is the complex conjugate of the residue of the pole of $F$ in $-i \omega_{0}$, and hence is positive semidefinite.

Let us consider now the case when $\omega_{0}=0$. We first assume that $G(s)$ has no pole at the origin. In this case $F(s)$ has no pole at the origin and $F(0)=0$, and $F(0)+F^{*}(0)=0 \geq 0$. If $G(s)$ has a simple pole at the origin, then $F(s)$ has no pole at the origin. Moreover, $G(s)=A / s+G_{1}(s)$ where $A$ is positive semidefinite. Thus $F(0)+F^{*}(0)=A+A^{\mathrm{T}}=2 A \geq$ 0 . If $G(s)$ has a double pole at the origin, we can write $G(s)=A_{2} / s^{2}+A_{1} / s+G_{1}(s)$, where $A_{2} \geq 0$. Hence $F(s)=$ $A_{2} / s+A_{1}+s\left(G_{1}(s)-G(\infty)\right)$. Since $G_{1}(s)$ is analytic in an open set containing the origin, also $A_{1}+s\left(G_{1}(s)-G(\infty)\right)$ is analytic in such an open set. Thus $F(s)$ has a simple pole at the origin and the corresponding residue $A_{2}$ is positive semidefinite.

Conversely, let $F(s)$ be symmetric, real rational and positive real matrix transfer function and $G(s) \stackrel{\text { def }}{=}(1 / s) F(s)+D$. As long as $D=D^{\mathrm{T}}$, the matrix $D$ is clearly irrelevant for the definition of symmetric negative imaginary transfer function, and it is sufficient to show that $G_{0}(s) \stackrel{\text { def }}{=}(1 / s) F(s)$ is symmetric negative imaginary. To this end, we first observe that $G_{0}(s)$ is a proper rational function and it is analytic in the open right half-plane. Moreover, $F(s)$ and $G_{0}(s)$ have the same poles with the possible exception of the pole at the origin. Let $\omega_{0}>0$ be given and assume that $s=i \omega_{0}$ is not a pole of $F(s)$. Then it is not a pole of $G_{0}(s)$. Moreover, $i\left[G_{0}\left(i \omega_{0}\right)-G_{0}^{*}\left(i \omega_{0}\right)\right]=i\left[\left(1 / i \omega_{0}\right) F\left(i \omega_{0}\right)+\left(1 / i \omega_{0}\right) F^{*}\left(i \omega_{0}\right)=\right.$ $\left(1 / \omega_{0}\right)\left[F\left(i \omega_{0}\right)+F^{*}\left(i \omega_{0}\right)\right]$ which is positive semidefinite because $\omega_{0}>0$ and $F$ is positive real. Let now $\omega_{0}>0$ be given and assume that $i \omega_{0}$ is a pole of $F(s)$ and hence of $G_{0}(s)$. Since $F(s)$ is positive real $i \omega_{0}$ is a simple pole of $F(s)$ and hence of $G_{0}(s)$. Moreover, the residue of $i \omega_{0}$ as a pole of $F(s)$ is a positive semidefinite matrix $A$. It is now evident that $\lim _{s \rightarrow i \omega_{0}}\left(s-i \omega_{0}\right) i G_{0}(s)=\left(1 / \omega_{0}\right) A$ which is clearly positive semidefinite.

The case of a pole at the origin is more delicate. If $F(0)=$ 0 then $G_{0}(s)$ does not have a pole at the origin and this concludes the proof. If $F(s)$ does not have a pole at the origin but $F(0) \neq 0$, then $G_{0}(s)$ has a simple pole at the origin and its residue is $F(0)$ which is positive semidefinite because $F$ is positive real. In the case when $F$ has a simple pole at the origin we use Lemma 3.2 and decompose $F(s)=$ $(1 / s) A+F_{1}(s)$ with $A$ symmetric and positive semidefinite and $F_{1}(s)$ positive real. Hence, $F_{1}(0)$ is positive semidefinite. Thus, $G_{0}(s)$ has a double pole at the origin, whose residue is $F_{1}(0) \geq 0$ and whose quadratic residue is $A \geq 0$.

Remark 3.1: As aforementioned, the difference between the above result and Lemma 1 in [17] is that we allow a double pole at the origin for negative imaginary transfer functions. This is very natural for two reasons: (i) positive real functions can have a single pole at the origin (the most common example being $F(s)=1 / s$ which is easily seen to be positive real) so that it is natural to allow a double pole at the origin for negative imaginary transfer functions. In fact, it is easily seen that $F(s)=1 / s$ satisfies Definition 1 in [17] and is therefore positive real (and this is extremely intuitive since $F(s)$ is the transfer function of a capacitor). Hence, in view of Lemma 1 in [17] we should have that $G(s)=1 / s^{2}$ is negative imaginary which is, again, intuitive. However, $G(s)=1 / s^{2}$ is not negative imaginary according to the Definition 2 in [17]. (ii) Since $G(s)=1 /\left(s^{2}+\varepsilon^{2}\right)$ is negative imaginary for any $\varepsilon \neq 0$, it is natural to require, by continuity, that also $G(s)=1 / s^{2}$ is negative imaginary. We observe that the above result is in line with Lemma 1 in [18], in which the definition of negative imaginary system was extended to include systems with a double pole at the origin.

\section{CONCLUDING REMARKS}

In this paper we presented a definition of symmetric negative imaginary system that hinges on properties of the transfer function matrix, and not on properties of a finitedimensional realisation. With the approach presented here even non rational transfer functions - which correspond to infinite-dimensional realisations - can turn out to be negative imaginary. We have then characterised negative imaginary rational transfer functions by an algebraic condition and established a connection between negative imaginary and positive real transfer functions. 


\section{REFERENCES}

[1] B.D.O. Anderson, and S. Vongpanitlerd. Network Analysis and Synthesis: a Modern Systems Theory Approach. Upper Saddle River, NJ: Prentice Hall, 1973.

[2] B. Brogliato, R. Lozano, B. Maschke and O. Egeland. Dissipative Systems Analysis and Control Theory and Applications. 2nd ed. New York: Springer-Verlag, 2007.

[3] P. Colaneri and A. Ferrante. Algebraic Riccati Equation and $J$-Spectral Factorization for $\mathscr{H}_{\infty}$ Filtering and Deconvolution. SIAM J. Contr. and Opt.. Vol. 45(1):123-145, 2006.

[4] A. Ferrante. On the Structure of the Solutions of Discrete-Time Algebraic Riccati Equation with Singular Closed-Loop Matrix. IEEE Trans. Automatic Control. Vol. AC-49(11):2049-2054, 2004.

[5] A. Ferrante. Positive real lemma: necessary and sufficient conditions for the existence of solutions under virtually no assumptions. IEEE Trans. Automat. Contr. Vol. AC-50(5):720-724, 2005.

[6] A. Ferrante. Minimal representations of continuous-time processes having spectral density with zeros in the extended imaginary axis. System \& Control Letters. Vol. 54(5):511-520, Apr. 2005.

[7] A. Ferrante, G. Marro, and L. Ntogramatzidis. A Parametrization of the Solutions of the Finite-Horizon LQ Problem with General Cost and Boundary Conditions. Automatica. Vol. 41:1359-1366, 2005.

[8] L. Ntogramatzidis, and A. Ferrante. The Generalised Discrete Algebraic Riccati Equation in Linear-Quadratic Optimal Control. $\mathrm{Au}$ tomatica. Vol. 49:471-478, DOI: 10.1016/j.automatica.2012.11.006, 2013.

[9] L. Ntogramatzidis, and A. Ferrante. On the solution of the Riccati differential equation arising from the LQ optimal control problem. Systems \& Control Letters. Vol. 59(2):114-121, 2010.

[10] A. Ferrante, and L. Ntogramatzidis. Employing the Algebraic Riccati Equation for a Parametrization of the Solutions of the Finite-Horizon LQ Problem: The Discrete-Time Case. Systems \& Control Letters. Vol. 54(7):693-703, 2005.

[11] A. Ferrante, and L. Ntogramatzidis, "Some new results in the theory of negative imaginary systems with symmetric transfer matrix function". Automatica, In press.

[12] A. Ferrante and M. Pavon. The Algebraic Riccati Inequality: Parametrization of Solutions, Tightest Local Frames and Generalized Feedback Matrices. Linear Algebra and its Applications. Vol. 292:187-206, 1999.

[13] A. Ferrante, M. Pavon, and S. Pinzoni. Asymmetric Algebraic Riccati Equation: A Homeomorphic Parametrization of the Set of Solutions. Linear Algebra and its Applications. Vol. 329:137-156, 2001

[14] M. A. Mabrok, A. G. Kallapur, I. R. Petersen and A. Lanzon. Stabilisation of conditional uncertain negative-imaginary systems using Riccati equation approach In Proceedings of the 20th International Symposium on Mathematical Theory of Networks and Systems (MTNS). Melbourne, July 9-13, 2012.

[15] A. Lanzon, and I.R. Petersen. Stability robustness of a feedback interconnection of systems with negative imaginary frequency response. IEEE Transactions on Automatic Control, AC-53(4):1042-1046, 2008.

[16] Z. Song, A. Lanzon, S. Patra, and I.R. Petersen. "Robust performance analysis for uncertain negative-imaginary systems". International Journal of Robust and Nonlinear Control, vol. 22(3), pp. 262-281, Feb 2012.

[17] M.A. Mabrok, A.G. Kallapur, I.R. Petersen, and A. Lanzon. "Stability analysis for a class of negative imaginary feedback systems including an integrator". In Proceedings of the $8^{\text {th }}$ Asian Control Conference 2011, Kaohsiung, Taiwan, May 15-18, 2011.

[18] M.A. Mabrok, A.G. Kallapur, I.R. Petersen, and A. Lanzon. "A stability result on the feedback interconnection of negative imaginary systems with poles at the origin". In Proceedings of the $2^{\text {nd }}$ Australian Control Conference 2012, pp. 98-103, Sydney, November 15-16, 2012.

[19] I. R. Petersen and A. Lanzon. Feedback control of negative imaginary systems. IEEE Control System Magazine, vol. 30, no. 5, pp. 54-72, 2010.

[20] Z. Song, A. Lanzon, S. Patra, and I.R. Petersen. Towards controller synthesis for systems with negative imaginary frequency response. IEEE Transactions on Automatic Control, AC-55:1506-1511, 2010.

[21] J. Xiong, I.R. Petersen, and A. Lanzon. A negative imaginary lemma and the stability of interconnections of linear negative imaginary systems. IEEE Transactions on Automatic Control, AC-55(10):23422347,2010
[22] J. Xiong, I.R. Petersen, and A. Lanzon. "On lossless negative imaginary systems". In Proceedings of the $7^{\text {th }}$ Asian Control Conference 2009, pp. 824-829, Hong Kong, Aug 2009.

[23] J. Xiong, I.R. Petersen, and A. Lanzon. "Finite frequency negative imaginary systems". IEEE Transactions on Automatic Control, AC57(11): 2917-2922, 2012.

[24] J. Xiong, I.R. Petersen, and A. Lanzon. On lossless negative imaginary systems. Automatica, 48:1213-1217, 2012. 